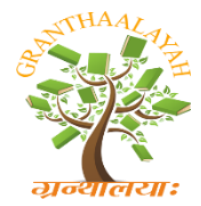

$$
\begin{gathered}
\text { INTERNATIONAL JOURNAL OF RESEARCH - } \\
\text { GRANTHAALAYAH } \\
\text { A knowledge Repository }
\end{gathered}
$$

Science

\title{
COMPARISON OF GLOBAL SEARCH OPTIMIZATION ALGORITHMS TO PERFORM THE VELOCITY ANALYSIS WITH A CONVERTED WAVE MOVEOUT EQUATION
}

\author{
Nelson Ricardo Coelho Flores Zuniga *1 \\ ${ }^{* 1}$ Departamento de Geofísica, Instituto de Astronomia, Geofísica e Ciências Atmosféricas, \\ Universidade de São Paulo, Brazil
}

\begin{abstract}
Even with previous works having studied about the accuracy and objective function of several nonhyperbolic multiparametric travel-time approximations for velocity analysis, they lack tests concerning different optimization algorithms and how they influence the accuracy and processing time. Once many approximations were tested and found the multimodal one which presented the best accuracy results, it is possible to perform a velocity analysis with different global search optimization algorithms. The minimization of the curve calculated with the converted wave moveout equation to the observed curve can be done for each optimization algorithm selected in this work. The travel-time curves tested here are the PP and PS reflection events coming from the interface of the top of an offshore ultra-deep reservoir. After the inversion routine have been performed, it is possible to define the processing time and the accuracy of each optimization algorithm for this kind of problem.
\end{abstract}

Keywords: Optimization Algorithms; Nonhyperbolic; Travel-Time; Seismic Inversion; Multiparametric.

Cite This Article: Nelson Ricardo Coelho Flores Zuniga. (2019). "COMPARISON OF GLOBAL SEARCH OPTIMIZATION ALGORITHMS TO PERFORM THE VELOCITY ANALYSIS WITH A CONVERTED WAVE MOVEOUT EQUATION.” International Journal of Research Granthaalayah, 7(6), 1-9. https://doi.org/10.29121/granthaalayah.v7.i6.2019.732.

\section{Introduction}

To solve the problem of the nonhyperbolicity during the velocity analysis step, many approximations where developed in previous works [2, 6, 18, 20, 25, 35]. However, even with a nonhyperbolic multiparametric travel-time approximation that is capable of control the nonhyperbolicity of a reflection event, the optimization can also be improved by selecting the most appropriate optimization algorithm to perform the inversion procedure. 
The comparison of nonhyperbolic multiparametric travel-time approximation was performed in many works recently [8, 38-42]. However, none of them considered different optimization algorithms to be tested. In this work, the multimodal approximation which presented the best set of results in previous works [18] was selected to be used; and five global search optimization algorithms were selected to perform the inversion. It was chosen for this tests an offshore ultradeep reservoir, due to its complexity in many situations that makes it able to force until limits of the nonhyperbolic approximation selected to be used.

With the inversion routine finished, it is possible to observe whether the nonhyperbolic multiparametric travel-time approximation presented a result reliable enough in this test. Thus, the main results reached testing the optimization algorithms is concerning the accuracy and the processing time.

\section{Materials and Methods}

Almost five decades after the creation of the hyperbola equation [7], Li and Yuan [18] proposed a converted wave moveout equation (Equation 1) to control the effects of the nonhyperbolicity by the parameter $\gamma$, previously studied by Li and Yuan [17] and based on the anisotropic parameters of Thomsen [33]. This nonhyperbolic multiparametric travel-time approximation considers the conversion point (CP) to control the nonhyperbolicity associated to the converted wave.

$t=\sqrt{t_{0}^{2}+\frac{x^{2}}{v^{2}}-\frac{(\gamma-1)}{\gamma v^{2}} \frac{(\gamma-1) x^{4}}{4 t_{0}^{2} v^{2}+(\gamma-1) x^{2}}}$

Where $\gamma$ is the ratio between the squared P-wave stacking velocity $v_{P 2}$ and the squared converted wave stacking velocity v_C2 (Equation 2 ).

$\gamma=\frac{v_{P 2}^{2}}{v_{C 2}^{2}}=\frac{\gamma_{e f f}\left(1+\gamma_{0}\right)}{\left(1+\gamma_{e f f}\right)}$

The relation $\gamma_{\text {eff }}$ is expressed by $\gamma_{\text {eff }}=\gamma_{2}^{2} / \gamma_{0}$, where $\gamma_{2}$ is the ratio between the stacking P-wave and stacking $\mathrm{S}$-wave, and $\gamma_{0}$ is the ratio between $\mathrm{P}$-wave velocity and $\mathrm{S}$-wave velocity which travel along the normal component.

Few years later Yuan [37] and Li [19] this approximation was also studied and, more than one decade later, it was strongly studied by the comparison of accuracy among several approximations and by the study of the topology of the objective function [38-43].

The main objective of the optimization is to reach the best option among a set of options, in other words, to maximize or to minimize a function selecting the input values and storing them to then find a maximum or minimum value [11]. In this work, it is aimed to reach the minimum point.

The processes of optimization usually generate an improvement in each interaction until reach the convergence in the minimum point of the function. However, several models can present more than one solution, due to its nonlinearity. This is common when an inversion problem is being 
solved, a situation which the focus is to obtain the best possible approximation of the parameters to be estimated.

The Adaptive Simulated Annealing (ASA) was developed for unconstrained optimization problems implemented for C. The Simulated Annealing algorithm depends on the magnitude, called as temperatures, of the parameters referred to as the cooling schedules where are decreasing sequences converge to zero sufficiently slow to allow the algorithm to avoid the local minimum region [24]. This algorithm was initially proposed to solve combinatorial problems [16] and after used for continuous and other problems [1, 5, 30]. Different than the conventional simulated annealing where it generates a probability density function with fatter tails than the Boltzmann distribution, this algorithm allows the possibility of scape from the local minimum region by considering points far away from the current iterate. Different temperatures of parameters are assigned for each variable and they are updated with the progression of the optimization.

The Response surface method (RSM) approximates an unknown function by a response surface, known as metamodel [4]. Any mismatch between the function and the metamodel is assumed to be caused by model error and not by the noise concerning the acquisition of the data. Response surfaces can be interpolating or not, and the former are obtained by minimizing the sum of the square deviations between the function and the metamodel at a number of points where the measurement of the function were reached [14]. After that, a common selection for noninterpolating surface is low-order polynomials are estimated by least regression on the experimental design [23]. It does not depend on the function used, but on the sampling technique [3].

The TOMLAB/EGO is an implementation of the EGO algorithm modified to handle both linear and nonlinear constraints [10]. The EGO algorithm is based on performing a space-filling experimental design and estimating the maximum likelihood for a model calculated and after the model is tested for consistency and accuracy [31]. A brunch-and-bound algorithm is used to optimize the expected improvement at a point. The expected improvement is defined, and the mean and standard deviation are compared to the predicted value and minimized from the minimum value [28]. Even with the possibility of the expected improvement function be reduced to a closedform expression, it can be strongly multimodal [15].

MCS (Multilevel Coordinate Search) is a Matlab implementation of the algorithm for global optimization of bound-constrained problems [26]. This algorithm is based on perform the partition of the search space into boxes with an evaluated base point. The global local search is balanced by a multilevel approach, according to which of each box is assigned a level $\mathrm{s}$ that is an increasing function of the number of times that the box war processed [12].

The TOMLAB/LGO is a TOMLAB solver implemented in Matlab. This kind of solver provides access to several derivative-free optimization solvers [10, 14]. The LGO (Local and Global Optimization) solver used here is a combination of global and local nonlinear solvers that implements a combination of Lipschitzian-based branch-and-bound with deterministic and stochastic local search [27-28]. 
Other optimization algorithms were considered, however for the similarities in the operations and in the results concerning the methods used here, some algorithms as SNOBFIT [13], PSWARM [36], CMA [9] and among others [29] were not used in this work.

The Model used in this work (Figure 1) is an offshore layered model with a carbonate reservoir $\left(V_{P}=4010 \mathrm{~m} / \mathrm{s}\right.$ and $V_{S}=2012 \mathrm{~m} / \mathrm{s}$ ) sealed by a salt structure composed by three layers (3rd, 4th and 5th layers) of salt with different physical properties (Table 1).
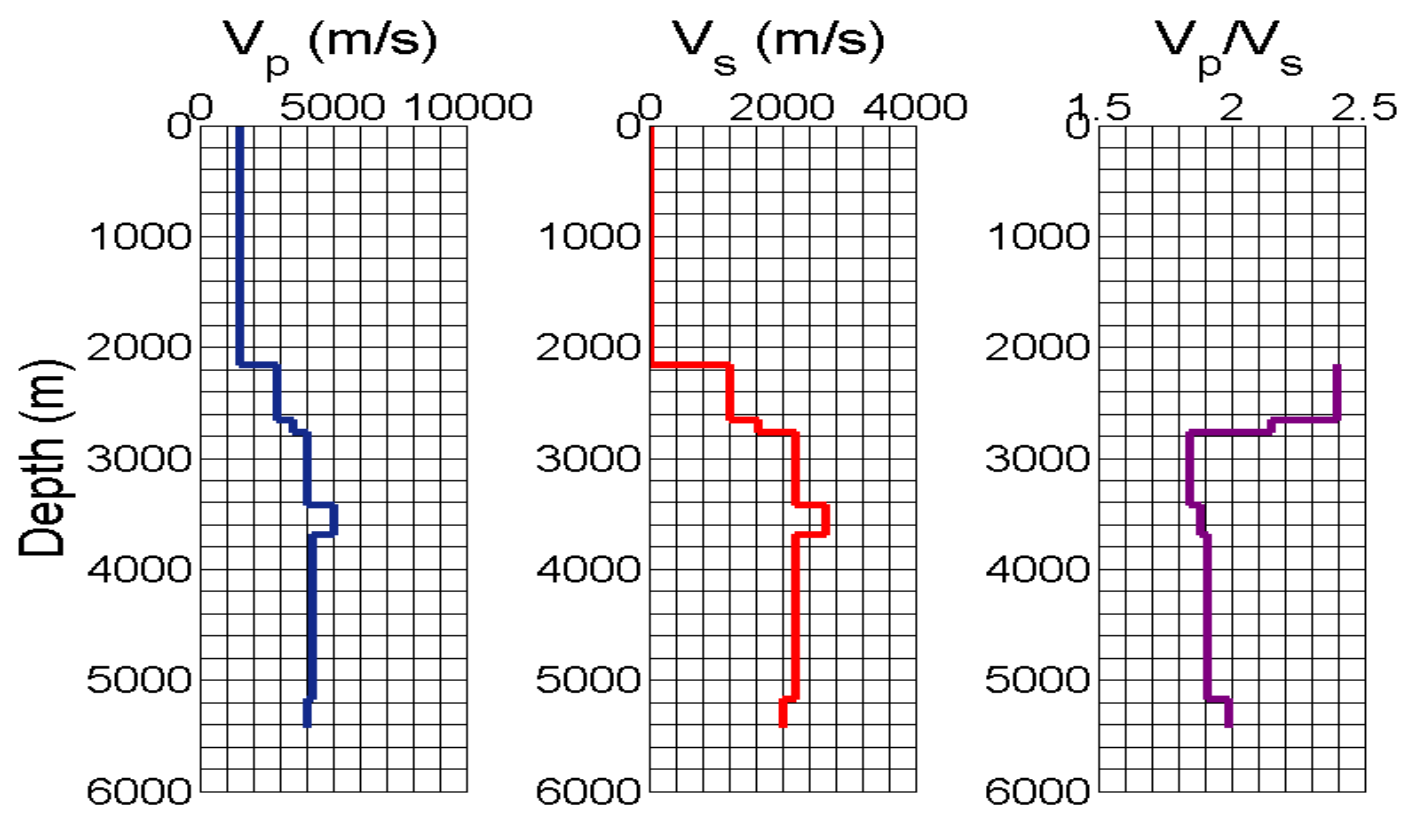

Figure 1: P-wave velocity $\left(V_{P}\right), \mathrm{S}$-wave velocity $\left(V_{S}\right)$ and $V_{P} / V_{S}$ ratio profiles of the Model.

Table 1: The parameters of the Model: Layer thickness $(\Delta \mathrm{z}), \mathrm{P}$-wave velocity $\left(V_{P}\right), \mathrm{S}$-wave velocity $\left(V_{S}\right)$ and $V_{P} / V_{S}$ ratio.

\begin{tabular}{|c|c|c|c|c|}
\hline Layer & $\begin{array}{c}\Delta \mathbf{z} \\
(\mathbf{m})\end{array}$ & $\begin{array}{c}\boldsymbol{V}_{\boldsymbol{P}} \\
(\mathbf{m} / \mathbf{s})\end{array}$ & $\begin{array}{c}\boldsymbol{V}_{\boldsymbol{S}} \\
(\mathbf{m} / \mathbf{s})\end{array}$ & $\begin{array}{c}\boldsymbol{V}_{\boldsymbol{P}} \\
\boldsymbol{V}_{\boldsymbol{S}}\end{array}$ \\
\hline Water & 2157 & 1500 & 0 & - \\
\hline 1 & 496 & 2875 & 1200 & 2.40 \\
\hline 2 & 108 & 3505 & 1628 & 2.15 \\
\hline 3 & 664 & 4030 & 2190 & 1.84 \\
\hline 4 & 262 & 5005 & 2662 & 1.88 \\
\hline 5 & 1485 & 4220 & 2210 & 1.91 \\
\hline
\end{tabular}

With the parameters, it is possible to generate the travel-time curves and the seismograms of the PP and PS reflection events by the ray tracing simulation [21-22, 34], showed in Figure 2. 

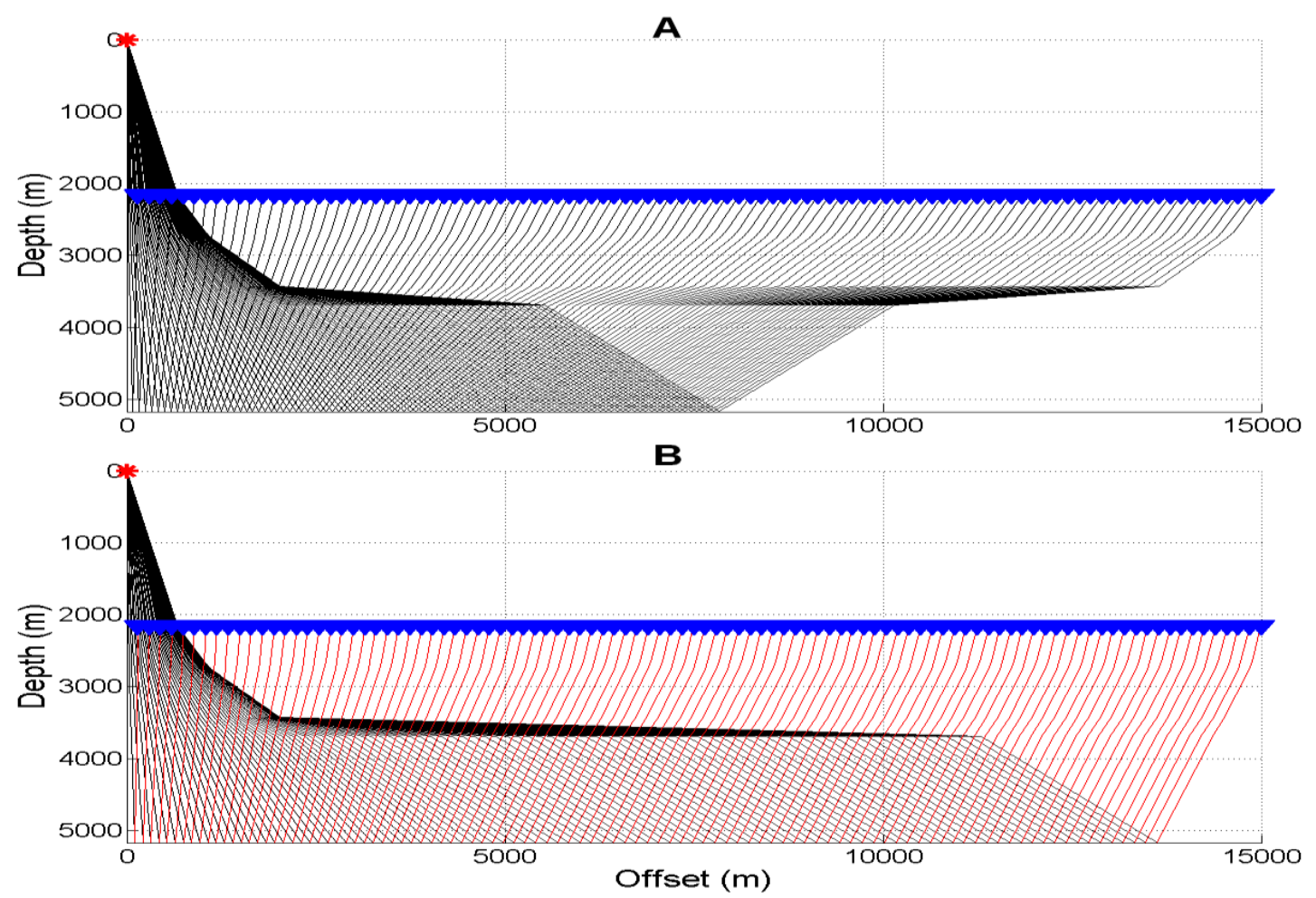

Figure 2: Ray tracing of the (A) PP wave reflection event and (B) PS wave reflection event of the Model.

\section{Results and Discussions}

The comparison of the residual travel-time analysis is performed observing the residual error between the observed curve and the calculated curve. Different than previous works where the comparison were performed considering several nonhyperbolic approximations [8, 38-43], here it is necessary to compare different optimization algorithms using the same nonhyperbolic multiparametric travel-time approximation, the one proposed by [18].

To compose each inversion routine it was performed one thousand inversions, for each optimization algorithm. One hundred inversion routines were performed to understand the stability of the problem. Once the problem showed to be stable, even being a multimodal problem, the mean accuracy and the mean processing time were computed to analyse which optimization algorithm showed the best performance.

In Figure 3A, it is possible to observe the residual travel-time error for the PP wave reflection event. In this case the ASA algorithm showed by far the highest error, while the other optimization algorithms showed more close results among them. However, the processing time (Table 2) of the ASA algorithm is considerably low. The RSM algorithm showed the second worst result with a relative low processing time. The TOMLAB/EGO algorithm presented a good result with an error limited in $0.02 \%$ and with a processing time a little higher than the RSM algorithm. The low error showed by the MCS algorithm is a considerably better result in comparison to the previous algorithms, and its processing time is a good result, even being significantly higher than the time presented by RSM and the TOMLAB/EGO algorithms. The lowest error was presented by 
TOMLAB/LGO algorithm, almost twice better than the TOMLAB/EGO and a little better than the MCS. However, the fifth algorithm used here presented a very high processing time.

Table 2: The mean processing time (in seconds) to perform the inversion routine (100 inversions) with each optimization algorithm for both reflection events.

\begin{tabular}{|c|c|c|}
\hline Algorithms & PP event & PS event \\
\hline ASA & 120.6 & 135.2 \\
\hline RSM & 162.5 & 172.9 \\
\hline TOMLAB/EGO & 178.3 & 189.7 \\
\hline MCS & 190.9 & 223.4 \\
\hline TOMLAB/LGO & 315.8 & 369.7 \\
\hline
\end{tabular}

The behavior of the algorithms for the PS wave reflection event (Figure 3B) is very similar to the showed by the conventional event. However, the difference of error is lower among the five algorithms, even with all of them presenting higher errors for the PS event, once this event is more complex due to its stronger nonhyperbolicity. The processing time (Table 2) is also higher than the one showed by the conventional event; however, the converted wave event has a similar proportionality of increase of the processing time. Concerning the residual error, TOMLAB/LGO presented again the best result, a little better than the MCS algorithm. With a significantly worse result, the TOMLAB/EGO algorithm showed the third best result. The second worst and the worst results were shown, respectively, by RSM and ASA algorithms.

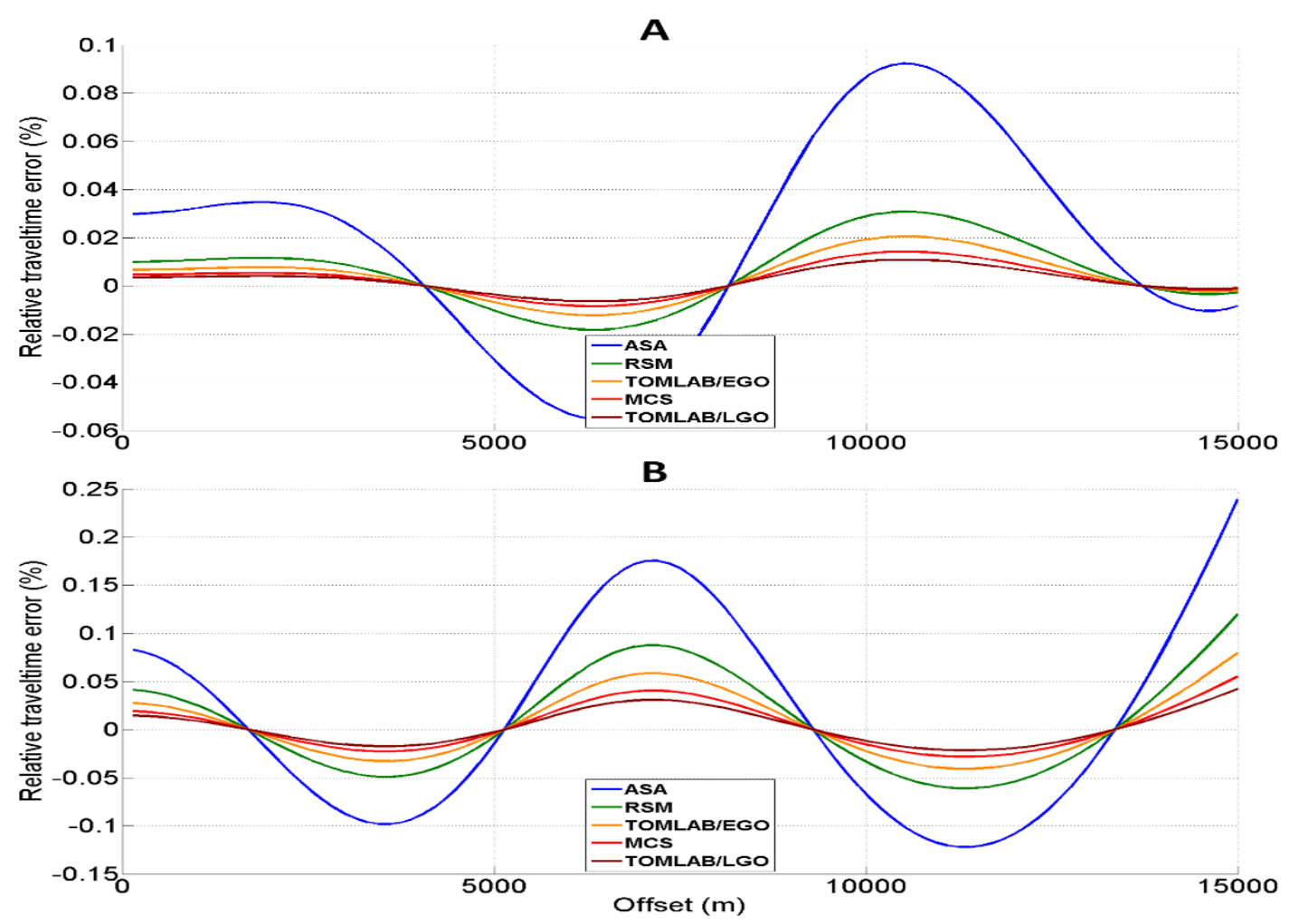

Figure 3: Relative errors in travel-time between the observed curve and the calculated curve with the approximation proposed by $\mathrm{Li}$ and Yuan [18] for each optimization algorithm for the (A) PP wave reflection event and (B) PS wave reflection event. 


\section{Conclusions and Recommendations}

The TOMLAB/LGO algorithm presented the best set of results concerning the residual travel-time error for both PP and PS reflection events. However, its processing time is much higher than the presented by the other algorithms. The MCS algorithm presented the second best set of results just a little worse than the presented by TOMLAB/LGO, but differently than the fifth algorithm, MCS showed a good processing time, not so higher than the other three. The TOMLAB/EGO and the RSM presented results considerably worse than the MCS even showing a little lower processing time. ASA algorithm presented the worst results with the highest error, event with the best processing times.

For this reason, the best trade-off relation was shown by MCS algorithm, with an acceptable processing time and a very low residual travel-time error what bring the most reliable results for this kind of problem.

\section{Acknowledgements}

This study was funded by Coordenação de Aperfeiçoamento de Pessoal de Nível Superior - Brasil (CAPES) (grant number 001) and by Conselho Nacional de Desenvolvimento Científico e Tecnológico - Brasil (CNPq).

\section{References}

[1] Aarts EHL, van Laarhoven PJM. Statistical cooling: A general approach to combinatorial optimization problems. Phillips Journal Research, 40, 1985, 193-226.

[2] Alkhalifah T, Tsvankin I. Velocity analysis for transversely isotropic Media. Geophysics, 60, 1995,1550-1566.

[3] Barros PA, Kirby MR, Mavris DN. Impact of sampling techniques selection on the creation of response surface models. SAE Transaction-Journal of Aerospace, 113, 2004, 1682-1693.

[4] Barton RR. Metamodeling: A state of the art review. Proceedings of the 1994 Winter Simulation Conference, Expanded Abstract, 1994, 237-244.

[5] Bélisle CJ, Romeijn HE, Smith RL. Hit-and-run algorithm for generating multivariate distributions. Mathematics of Operations Research, 18, 1993, 255-266.

[6] Blias E. Long-offset NMO approximations for a layered VTI model: Model study. 79th Annual International Meeting, Society of Exploration Geophysics, Expanded Abstract, Houston, 2009, 3745-3749.

[7] Dix CH. Seismic velocities from surface measurements. Geophysics, 20, 1955, 68-86.

[8] Golikov P, Stovas A. Accuracy comparison of nonhyperbolic moveout approximations for qPwaves in VTI media. Journal of Geophysics and Engineering, 9, 2012, 428-432.

[9] Hansen N. The CMA evolution strategy: A computing review. Towards a New Evolutionary Computation, 2006, 75-102.

[10] Holmström K, Quttineh NH, Edvall MM. An adaptive radial basis algorithm (ARBF) for expensive black-box mixed-integer constrained global optimization. Optimization and Engineering, 9, 2008, 311-339.

[11] Horst R, Pardalos PM, Thoai NV. Introduction to global optimization. 2nd ed. Dordrecht: Kluwer Academic Publusher, 2000, 354.

[12] Huyer W, Neumaier A. Global optmization by multilevel coordinate search. Journal of Global Optimization, 14, 1999, 331-355. 
[13] Huyer W, Neumaier A. SNOBFIT - Stable Noisy optimization by branch and fit. ACM Transactions on Mathematical Software, 35, 2008, 1-25.

[14] Jones DR. A taxonomy of the global optimization methods based on response surface. Journal of Global Optimization, 21, 2001, 345-383.

[15] Jones DR, Schonlau M, Welch J. Efficient global optimization of expensive black-box functions. Journal of Global Optimization, 13, 1998, 455-492.

[16] Kirkpatrick S, Gelatt CD, Vecchi P. Optimization by simulated annealing. Science, 220, 1983, 671680.

[17] Li XY, Yuan J. Converted-waves moveout and parameter estimation for transverse isotropy. 61st EAGE Conference, Expanded Abstract, 1999, 4-35.

[18] Li XY, Yuan J. Converted wave imaging in inhomogeneous, anisotropic media: Part I. Parameter estimation. 63rd EAGE Conference, Amsterdam, The Netherlands., Expanded Abstract, 2001, 109.

[19] Li XY, Converted-wave moveout analysis revisited: The search for a standard approach. 73rd Annual International Meeting, Society of Exploration Geophysics, Expanded Abstract, 2003, 805808.

[20] Malovichko AA. A new representation of the traveltime curve of reflected waves in horizontally layered media. Applied Geophysics (in Russian), 91, 1978, 47-53.

[21] Margrave GF. New seismic modelling facilities in Matlab. CREWES Research Report, 12, 2000.

[22] Margrave GF. Numerical methods explorations seismology with algorithms in Matlab. CREWES Research Report, 2003.

[23] Matheron G. Principles of geostatistics. Economic Geology, 58, 1967, 1246-1266.

[24] Metropolis N, Rosenbluth AW, Rosenbluth MN, Teller AH, Teller E. Equation of state calculations by fast computing machine. The Journal of Chemical Physics, 21, 1953, 1087-1092.

[25] Muir F, Dellinger J. A practical anisotropic system, in SEP-44. Stanford Exploration Project, 1985, 55-58.

[26] Neumaier A, Shcherbina R, Huyer W, Vinkó T. A comparison of complete global optimization solvers. Mathematical Programming, 103, 2005, 335-356.

[27] Pintér JD. Global optimization in action: Continuous and Lipschitz optimization. Algorithms, Implementations and Applications, Kluwer Academic Publisher, 1995, 422.

[28] Pintér JD, Holmström K, Göran AO, Edvall MM.. User's Guide for TOMLAB/LGO. TOMLAB Optimization, 2006.

[29] Rios LM, Sahinidis NV. Derivative-free optimization: A review of algorithms and comparison of softwares implementations. Journal of Global Optimization, 56, 2013, 1247-1293.

[30] Romeo F, Sangiovani-Vincentelli A. A theoretical framework for simulated annealing. Algorithmica, 6, 1991, 302-345.

[31] Schonlau M. Compute Experiments and Global Optimization. PhD Thesis, University of Waterloo, 1997, 131.

[32] Telraky T, Sotirov R. Multi-start approach to global conic optimization. ISE Archives of Working Papers, 2010.

[33] Thomsen L. Weak elastic anisotropy. Geophysics, 51, 1986, 1954-1966.

[34] Thorbecke JW, Draganov D. Finite-difference modeling experiment for seismic interferometry. Geophysics, 76, 2012, H1-H18.

[35] Ursin B, Stovas A. Traveltime approximations for a layered transversely isotropic medium. Geophysics, 71, 2006, 23-33.

[36] Vaz AIF, Vicente LN. A particle swarm pattern search method for bound constrained global optimization. Journal of Global Optimization, 39, 2007, 197-219.

[37] Yuan J, Li XY. Comverted wave anisotropic parameter estimation from conversion point. 64th EAGE conference, Expanded Abstract, 2002, 253.

[38] Zuniga NRCF. Análise comparativa de aproximações não-hiperbólicas dos tempos de trânsito de dados sísmicos multicomponente utilizando tecnologia OBN. Master's Thesis, Universidade de São Paulo, Brazil, 2017, 86. 
[39] Zuniga NRCF, Bokhonok O, Diogo LA. Comparison of nonhyperbolic travel-time approximations for multicomponent seismic data. 14th SBGf Congress, Expanded Abstract, Rio de Janeiro, Brazil, 2015, 1176-1181.

[40] Zuniga NRCF, Molina EC, Prado RL. Inversion of multicomponent seismic data for VTI medium using the globalized Nelder-Mead optimization algorithm. 3th EAGE/SBGf Workshop, Expanded Abstract. Rio de Janeiro, Brazil, 2016a.

[41] Zuniga NRCF, Molina EC, Prado RL. Inversion of multicomponent seismic data of the Santos Basin. Far East Hydrocarbons, Expanded Abstract, 2016b.

[42] Zuniga NRCF, Molina EC, Prado RL. Comparison of travel-time approximations for unconventional reservoirs from Santos Basin, Brazil. Brazilian Journal of Geophysics, 35, 2017, 271-286.

[43] Zuniga NRCF, Ribeiro FB, Priimenko VI. Relation between the model and the topography of the objective function in a velocity analysis using a nonhyperbolic multicomponent travel-time approximation. Brazilian Journal of Geophysics, 36(4), 2018, 1-10.

*Corresponding author.

E-mail address: nelson.zuniga @iag.usp.br 